\title{
The MultiSite Spectroscopic Telescope campaign: 2 m spectroscopy of the V361 Hya variable PG 1605+072
}

\author{
S. J. O’ Toole ${ }^{1,2}$, U. Heber ${ }^{1}$, C. S. Jeffery ${ }^{3}$, S. Dreizler ${ }^{4,5}$, S. L. Schuh ${ }^{4,5}$, V. M. Woolf ${ }^{3,6}$, S. Falter ${ }^{1,8}$, \\ E. M. Green ${ }^{7}$, B.-Q. For ${ }^{7}$, E. A. Hyde ${ }^{7}$, H. Kjeldsen ${ }^{9}$, T. Mauch ${ }^{2}$, and B. A. White ${ }^{7}$ \\ 1 Dr. Remeis-Sternwarte, Astronomisches Institut der Universität Erlangen-Nürnberg, Sternwartstr. 7, \\ 96049 Bamberg, Germany \\ e-mail: otoole@sternwarte.uni-erlangen.de \\ 2 School of Physics, University of Sydney, NSW 2006, Australia \\ 3 Armagh Observatory, College Hill, Armagh BT61 9DG, UK \\ ${ }^{4}$ Institut für Astrophysik, Universität Göttingen, Friedrich-Hund-Platz 1, 37077 Göttingen, Germany \\ 5 Institut für Astronomie und Astrophysik, Universität Tübingen, Sand 1, 72076 Tübingen, Germany \\ ${ }^{6}$ Department of Physics, University of Nebraska at Omaha, 6001 Dodge St., Omaha NE 68182, USA \\ 7 Steward Observatory, University of Arizona, 933 North Cherry Avenue, Tucson, AZ 85721, USA \\ 8 Max-Planck-Institut für Astronomie, Königstuhl 17, 69117 Heidelberg, Germany \\ 9 Teoretisk Astrofysik Center, Danmarks Grundforskningsfond, 8000 Aarhus C, Denmark
}

Received 2 May 2005 / Accepted 25 May 2005

\begin{abstract}
We present results and analysis for the $2 \mathrm{~m}$ spectroscopic part of the MultiSite Spectroscopic Telescope (MSST) campaign undertaken in May/June 2002. The goal of the project was to observe the pulsating subdwarf B star PG $1605+072$ simultaneously in velocity and photometry and to resolve as many of the $>50$ known modes as possible, which will allow a detailed asteroseismological analysis. We have obtained over $150 \mathrm{~h}$ of spectroscopy, leading to an unprecedented noise level of only $207 \mathrm{~m} \mathrm{~s}^{-1}$. We report here the detection of 20 frequencies in velocity, with two more likely just below our detection threshold. In particular, we detect 6 linear combinations, making PG 1605+072 only the second star known to show such frequencies in velocity. We investigate the phases of these combinations and their parent modes and find relationships between them that cannot be easily understood based on current theory. These observations, when combined with our simultaneous photometry, should allow asteroseismology of this most complicated of sdB pulsators.
\end{abstract}

Key words. stars: individual: PG 1605+072 - stars: oscillations

\section{Introduction}

Hot subdwarf B (sdB) stars are generally associated with models of extreme Horizontal Branch (EHB) stars, i.e. they burn helium in their cores, and have hydrogen envelopes that are too thin to sustain nuclear fusion (e.g. Heber 1986). Canonical EHB models show the stars to have a very narrow mass range around $\sim 0.48 M_{\odot}$, and envelope masses $<0.02 M_{\odot}$ (Dorman et al. 1993). How an sdB arrives at this configuration is one of the many unresolved questions regarding these stars. One of the most promising formation mechanisms is binary interaction or the merger of two low-mass white dwarfs (e.g. Han et al. 2003).

The discovery of short-period $(P<10 \mathrm{~min})$ multimode pulsations in some sdBs provides an excellent opportunity to probe the interiors of these stars using the tools of asteroseismology, thereby answering some of the above questions. Using small telescopes (typically 1-2 m) and examining hundreds of sdBs has yielded around 30 of these pulsators, each with amplitude $<50$ mmag (for a review of these objects, now known as V361 Hya stars, see Kilkenny 2002). The periods suggest that the stars are $p$-mode pulsators, but asymptotic theory cannot be applied to the analysis of their frequency spectra. The possibility of using oscillations to probe the interiors of sdBs received another boost after the discovery of pulsations with periods of $45 \mathrm{~min}-2 \mathrm{~h}$ (Green et al. 2003). The much longer period pulsations found in these stars indicate they are $g$-modes. The stars are typically cooler than the $p$-mode pulsators. Theoretical modelling has found that the pulsations in both groups may be driven by an opacity bump due to ionisation of iron (and other iron-group elements) (Charpinet et al. 2001; Fontaine et al. 2003).

There has been some limited success in asteroseismic studies of pulsating sdBs. Brassard et al. (2001) detected 13 pulsation frequencies in PG 0014+067, matched these with a model and subsequently inferred the star's fundamental stellar parameters. The models used require that modes with $l=3$ need to be excited to explain the density of modes observed. Geometric cancellation was believed to exclude observations of 
such high degree modes in intensity, although recently Jeffery et al. (2004) claimed $l=4$ modes using high-speed, multicolour photometry on the William Herschel telescope. Another way to test this idea is by measuring stellar surface motions, which suffer less from cancellation. An attempt to do this has been made for several stars, including PG 1605+072 (see below for more details), PB 8783 and KPD 2109+4401 (Jeffery \& Pollacco 2000), KPD 1930+2752 (Woolf et al. 2002a), PG 1336-018 (Woolf et al. 2003) and PG 1325+101 (Telting \& Østensen 2004). Until now, though, it has not been possible to measure enough velocity and intensity amplitudes simultaneously to test whether $l=3$ modes in sdBs can be measured in intensity.

PG 1605+072 was discovered to pulsate by Koen et al. (1998). The star has the longest periods (up to $9 \mathrm{~min}$ ) and the highest amplitudes (up to $\sim 60 \mathrm{mmag}$ ) of the sdBV (or V361 Hya) stars currently known. It was clear from the discovery observations that the star showed great asteroseismological potential, so a multisite photometric campaign was organised to observe the star continuously for two weeks. The results can be found in Kilkenny et al. (1999), who detected more than 50 frequencies in the amplitude spectrum. It is difficult to say how many of these are independent normal modes of oscillation as there is evidence for amplitude variability of timescales of at most a few months. Kawaler (1999) found a possible model match to the 5 highest amplitude frequencies measured by Kilkenny et al., suggesting that the star has an equatorial rotation velocity of $130 \mathrm{~km} \mathrm{~s}^{-1}$. This was given support from spectral analysis by Heber et al. (1999), who determined $v \sin i=39 \mathrm{~km} \mathrm{~s}^{-1}$ from high-resolution spectroscopy, suggesting an inclination angle of $\sim 17^{\circ}$. This makes PG $1605+072$ the most rapidly rotating and apparently single sdB known. Heber et al. also found $T_{\text {eff }}=32300 \mathrm{~K}, \log g=5.25, \log (\mathrm{He} / \mathrm{H})=$ -2.53 and measured metal abundances; the low $\log g$ implies the star is also quite evolved, and has moved away from the EHB.

Because of its long periods, high amplitudes and relative brightness, PG $1605+072$ is an ideal target for rapid spectroscopy in comparison with other pulsating sdBs. Velocity variations were first detected by O'Toole et al. (2000), at frequencies corresponding to those found in photometry and with amplitudes of up to $14 \mathrm{~km} \mathrm{~s}^{-1}$. A more detailed analysis of these and follow-up observations was presented by O'Toole et al. (2002). Other studies of PG 1605+072 include Woolf et al. (2002b), who used $4 \mathrm{~m}$ class telescopes to achieve better velocity accuracy than both O'Toole et al. (2000) and O'Toole et al. (2002), but were hampered by timing problems and a short temporal baseline. A study of Balmer line indices by O'Toole et al. (2003) measured effective temperature and gravity changes, producing a new set of observables that may be used for mode identification. Finally, as a feasibility study, Falter et al. (2003) conducted the first simultaneous multicolour photometry and velocity observations. These studies suffered from either no (or little) simultaneous photometry, poor frequency resolution, or a combination of both. With this in mind, we organised a multisite coordinated spectroscopic campaign to observe PG $1605+072$ with medium resolution spectrographs on $2 \mathrm{~m}$ and $4 \mathrm{~m}$ class telescopes and for as long
Table 1. Table of MSST spectroscopic observations. KPNO = Steward $2.3 \mathrm{~m}$ on Kitt Peak; LS = Danish $1.54 \mathrm{~m}$ on La Silla; $\mathrm{SSO}=2.3 \mathrm{~m}$ on Siding Spring; LP = Nordic Optical Telescope $(2.56 \mathrm{~m})$ on La Palma.

\begin{tabular}{|c|c|c|c|c|}
\hline UT Date & Where & $\begin{array}{c}\text { UT } \\
\text { start }\end{array}$ & $\begin{array}{c}\text { UT } \\
\text { finish }\end{array}$ & $\begin{array}{l}\text { No. of } \\
\text { spectra }\end{array}$ \\
\hline $19 / 05 / 2002$ & KPNO & $03: 58$ & $11: 12$ & 344 \\
\hline $20 / 05 / 2002$ & KPNO & 04:04 & $11: 40$ & 434 \\
\hline $21 / 05 / 2002$ & KPNO & $03: 35$ & $11: 18$ & 440 \\
\hline $22 / 05 / 2002$ & LS & $02: 30$ & $08: 43$ & 315 \\
\hline $22 / 05 / 2002$ & KPNO & $03: 24$ & $11: 19$ & 455 \\
\hline $23 / 05 / 2002$ & LS & $02: 15$ & $08: 35$ & 348 \\
\hline $23 / 05 / 2002$ & KPNO & $03: 28$ & $11: 25$ & 452 \\
\hline $24 / 05 / 2002$ & KPNO & $03: 16$ & $11: 29$ & 470 \\
\hline $25 / 05 / 2002$ & SSO & $14: 56$ & $17: 56$ & 160 \\
\hline $26 / 05 / 2002$ & SSO & $10: 43$ & $17: 15$ & 360 \\
\hline $27 / 05 / 2002$ & SSO & $11: 14$ & $17: 56$ & 360 \\
\hline $13 / 06 / 2002$ & LS & $00: 54$ & $07: 11$ & 202 \\
\hline $14 / 06 / 2002$ & LS & $00: 36$ & 07:04 & 334 \\
\hline $15 / 06 / 2002$ & LS & $00: 44$ & $06: 51$ & 340 \\
\hline $16 / 06 / 2002$ & LS & $00: 32$ & $06: 45$ & 318 \\
\hline $17 / 06 / 2002$ & LS & $00: 56$ & $06: 50$ & 334 \\
\hline $18 / 06 / 2002$ & LP & $21: 36$ & $04: 34$ & 460 \\
\hline $18 / 06 / 2002$ & LS & $00: 32$ & $06: 48$ & 317 \\
\hline $19 / 06 / 2002$ & LP & $20: 53$ & 03:14 & 400 \\
\hline $19 / 06 / 2002$ & LS & $00: 36$ & $06: 43$ & 326 \\
\hline $20 / 06 / 2002$ & LP & $20: 54$ & $04: 28$ & 520 \\
\hline 20/06/2002 & LS & $00: 24$ & 06:39 & 333 \\
\hline $21 / 06 / 2002$ & LP & $20: 52$ & $04: 26$ & 500 \\
\hline $21 / 06 / 2002$ & LS & 01:38 & $06: 20$ & 260 \\
\hline $22 / 06 / 2002$ & LP & $22: 12$ & $04: 21$ & 435 \\
\hline $22 / 06 / 2002$ & LS & $00: 27$ & $06: 31$ & 326 \\
\hline $23 / 06 / 2002$ & LP & $21: 43$ & 04:18 & 435 \\
\hline $24 / 06 / 2002$ & LP & $20: 48$ & 03:09 & 430 \\
\hline $25 / 06 / 2002$ & LP & $20: 49$ & $04: 25$ & 540 \\
\hline total & & & & 10892 \\
\hline
\end{tabular}

as possible. To complement these observations, we obtained simultaneous photometry using $1 \mathrm{~m}$ class telescopes. The project was greatly aided by observations during the $X \operatorname{cov} 22$ campaign of the Whole Earth Telescope.

In this paper we present first spectroscopic results from the MultiSite Spectroscopic Telescope (MSST) campaign which observed PG 1605+072 in May/June 2002 (see Heber et al. 2003). We have obtained $151 \mathrm{~h}$ of time-resolved spectroscopy which was taken over 38 nights using four $2 \mathrm{~m}$ telescopes. We increase the number of detected frequencies in velocity by a factor of two. The work represents the first step in understanding one of the most complicated sdB pulsators. Two further papers will be published on observations from this campaign: results from $4 \mathrm{~m}$ telescopes (spectroscopy) and results from photometry (both in preparation).

\section{Observations}

The MSST spectroscopic campaign was divided into two parts, shown in Table 1. The first part (19-27/05/2002) overlapped 
Table 2. Instrumentation used in the $2 \mathrm{~m}$ spectroscopy part of the MSST campaign.

\begin{tabular}{|c|c|c|c|c|c|c|c|c|c|c|}
\hline Observatory & $\begin{array}{c}D \\
(\mathrm{~m})\end{array}$ & $\begin{array}{c}\text { Grating } \\
\text { (lines/mm) }\end{array}$ & $\begin{array}{c}\text { Dispersion } \\
(\AA / \text { pix })\end{array}$ & $\begin{array}{l}\text { Slit } \\
\left({ }^{\prime \prime}\right)\end{array}$ & $\begin{array}{c}\text { Resolution } \\
(\AA)\end{array}$ & $\begin{array}{l}\text { CCD size } \\
\text { (pix) }\end{array}$ & Binning & $\begin{array}{c}\lambda \text { range } \\
(\AA)\end{array}$ & $\begin{array}{l}t_{\exp } \\
(\mathrm{s})\end{array}$ & $\begin{array}{l}t_{\text {dead }} \\
\text { (s) }\end{array}$ \\
\hline Steward/KPNO & 2.3 & 832 & 0.707 & 1.5 & 1.8 & $1200 \times 800$ & $1 \times 3$ & $3686-4534$ & 45 & 12 \\
\hline Siding Spring & 2.3 & 600 & 1.1 & 2.0 & 2.2 & $1752 \times 532$ & $1 \times 1$ & $3647-5047$ & 45 & 10 \\
\hline Danish/ESO & 1.54 & DFOSC\#6 & 1.65 & 1.5 & 5.9 & $2 \mathrm{~K} \times 4 \mathrm{~K}$ & $3 \times 2$ & $3648-5147$ & $35 / 45$ & 17 \\
\hline NOT & 2.56 & ALFOSC\#3 & 2.3 & 0.75 & 9.3 & $2 \mathrm{~K} \times 2 \mathrm{~K}$ & $3 \times 2$ & 3039-6669 & 35 & 17 \\
\hline
\end{tabular}

with the photometric observations of the MSST campaign, while the second part (13-25/06/2002) took place about 2 weeks afterwards. We were granted time on four 2 m-class telescopes: the Steward Observatory $2.3 \mathrm{~m}$ on Kitt Peak (6 nights); the Danish 1.54 at La Silla (8 nights in ESO time and 10 nights in Danish time); the $2.3 \mathrm{~m}$ Advanced Technology Telescope at Siding Spring Observatory (8 nights); and the $2.56 \mathrm{~m}$ Nordic Optical Telescope at La Palma (8 nights).

Previous spectroscopic observations of PG $1605+072$ with $2 \mathrm{~m}$ telescopes proved the feasibility of a study of velocity variations, so the main focus of this part of the MSST was to maximise the length and improve the coverage compared with studies such as O'Toole et al. (2002).

\subsection{Instrumentation}

In Table 2 we give the details of the instrumental setup for each telescope. To ensure accurate timing, multiple shutter tests were conducted at KPNO and SSO. This was a necessary check since the UT written in the image header does not correspond to the time the shutter actually opened for some systems. At the former telescope, correction of the UT in the image headers was required, giving an accuracy to within a few tenths of a second, while at the latter, no corrections were required as the times were accurate to better than one second. From our previous experience at La Silla and La Palma we know the timing of these telescopes to be accurate to less than one second. To improve readout times, the CCDs were windowed. This could not improve the size of the dead time of DFOSC and ALFOSC however. They are large because before each exposure the systems perform checks of both the filter and grism wheels. An override of this feature does not seem to be possible.

\subsection{Reductions}

The observations described above were reduced with IRAF, using the standard bias subtraction, flat fielding, aperture tracing, sky correction and spectrum extraction routines. Wavelengths were calibrated using arc spectra that were taken roughly once per hour. This was done to minimise spectrograph drifts from the velocity curve and therefore reduce the $1 / f$ noise component in the final amplitude spectrum (although see below). At the NOT arc spectra were taken at the beginning and end of each night only, and drifts needed to be removed by hand.

All velocities were determined by cross-correlating each spectrum with a nightly template, created by median filtering that night's spectra. For observations from the Steward Observatory $2.3 \mathrm{~m}$, the templates were corrected to rest wavelengths using an iterative procedure, however this was not done for the other observations since it was deemed that the actual velocity shift is smaller than the spectral resolution; the effect of not making this correction does not appear to be significant. The cross correlations were done in IRAF using a doubleprecision version of the package fxcor ${ }^{1}$. Unlike previous velocity studies (e.g. O'Toole et al. 2002), where Balmer lines were examined individually, with the resulting velocity curves combined, the entire spectrum was cross-correlated in order to achieve the highest accuracy.

\subsection{Velocities}

Our resulting velocity curve is shown in the top panel of Fig. 1. In the case of DFOSC and ALFOSC, both grism spectrographs, regular arc spectra reduce the drift, but by no means remove it completely, which is seen as apparently high scatter, particularly on the right side of the figure. In contrast, the drift in the velocities determined from data taken with grating spectrographs (Steward and SSO $2.3 \mathrm{~m}$ telescopes) is almost completely removed. Similar drifts are seen in other time-series ALFOSC spectra (e.g. observations of another V361 Hya star, PG $1325+101$ by Telting \& Østensen 2004). The drifts, and their removal, are discussed in more detail below.

The spectral windows of the two parts of the campaign and the whole campaign are shown in Figs. 2 and 3, respectively. These serve as a guide to the aliasing we can expect in the amplitude spectrum. It can be seen from Fig. 2 that the coverage during two halves of the campaign was approximately the same, although the frequency resolution of the second part is better than the first. The result of this is that the spectral window of the entire campaign is more or less the same as the two halves, except for the introduction of fine structure caused by their two week separation, which is shown in the top panel of Fig. 3. The separation between the main alias peaks corresponds to one cycle-per-day (measured to be $11.67 \mu \mathrm{Hz}$ rather than $11.57 \mu \mathrm{Hz}$ ), while the small separation (shown in the inset) is $\sim 0.38 \mu \mathrm{Hz}$. The latter alias corresponds to the difference between midpoints in the two parts of the campaign, rather than the length of time between them. These aliases must be considered when conducting any frequency analysis.

Because the quality of the data varies from night to night, and even during the night, we have assigned weights to each velocity based on the velocity error determined by fxcor. Studies by O'Toole et al. $(2000,2002)$ used the inverse square of the local rms scatter of each night as weights - the advantage of using the velocity error is that individual points are considered,

\footnotetext{
${ }^{1}$ Available at http://iraf.noao.edu/scripts/extern/rvx.pl
} 

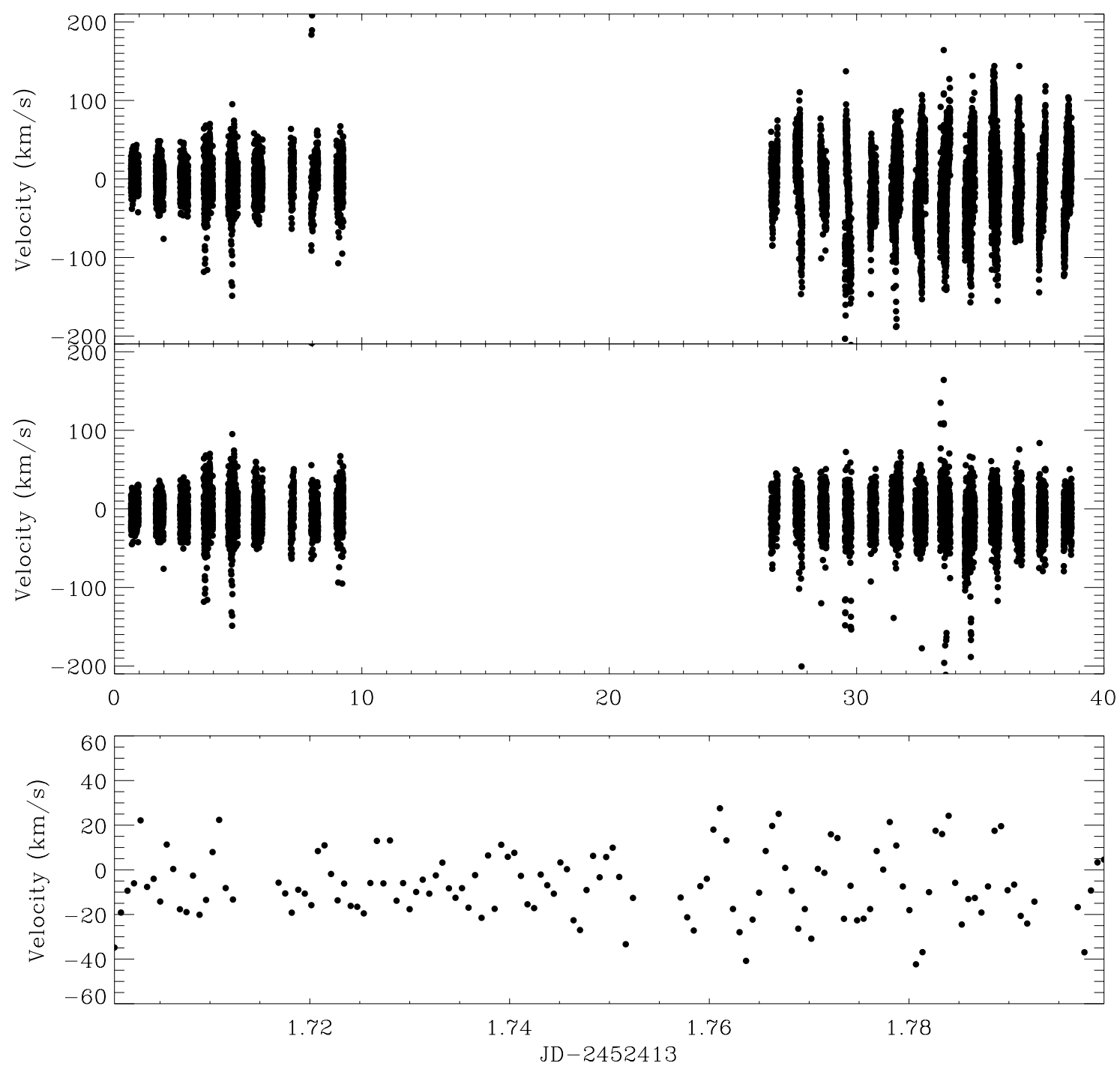

Fig. 1. Velocity curve of the entire $2 \mathrm{~m}$ telescope part of the MSST campaign before (top) and after (middle) removal of slow trends. A section of the curve from the second night of observations (20 May 2002) at Steward Observatory is also shown (bottom); the variations at $\sim 480 \mathrm{~s}$ are evident around 1.76 days.

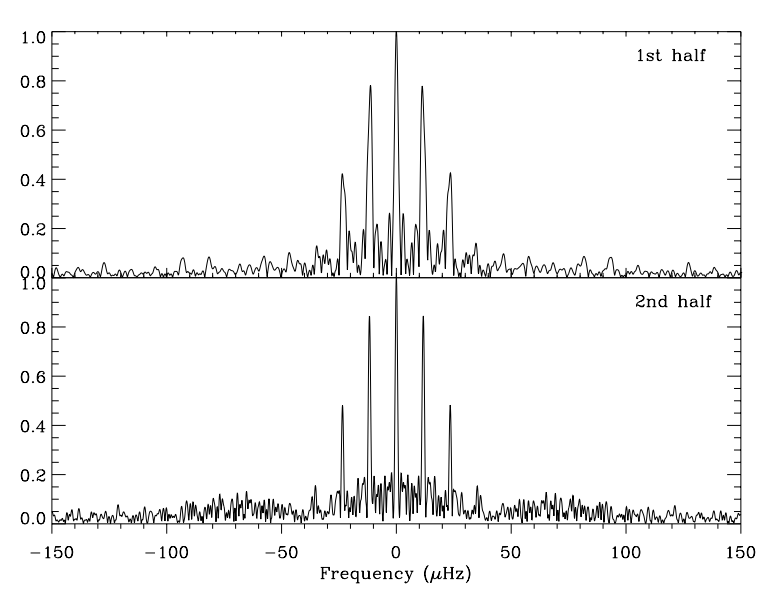

Fig. 2. Spectral windows of the two halves of the $2 \mathrm{~m}$ spectroscopic campaign. The top panel represents the window for 19-27/05/2002, while the bottom represents the window for 13-25/06/2002.

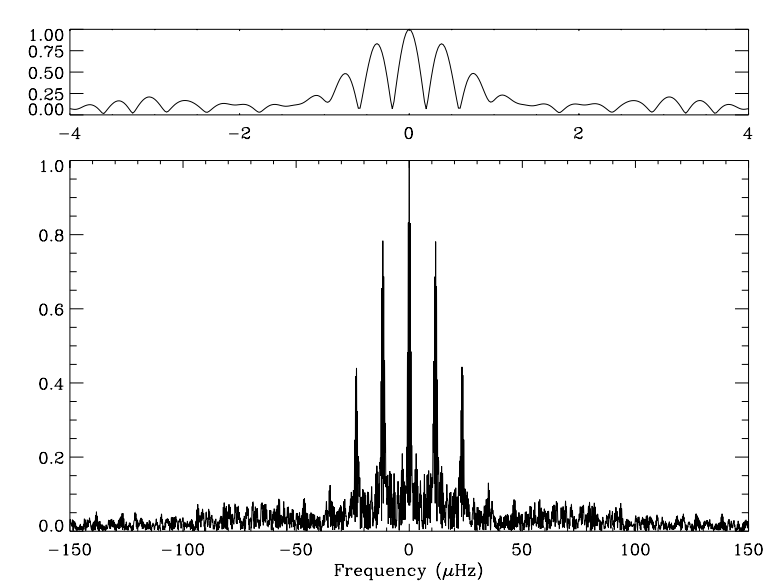

Fig. 3. Spectral window of all $2 \mathrm{~m}$ spectroscopic observations. The top panel shows fine structure in the central peak caused by the 2 week gap in observations. The spacing between the alias peaks is $\sim 0.38 \mu \mathrm{Hz}$. 


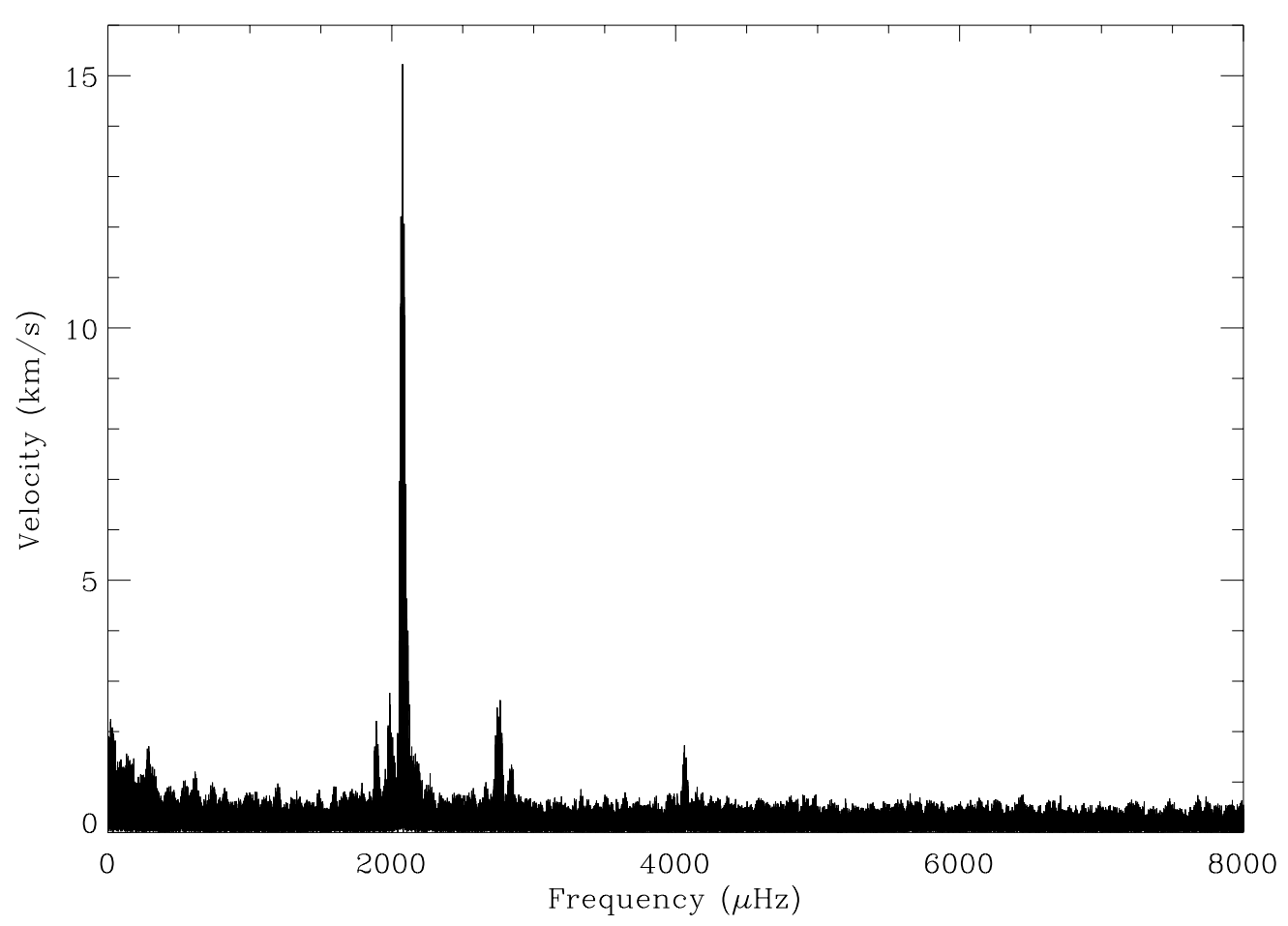

Fig. 4. The velocity amplitude spectrum based on $2 \mathrm{~m}$ telescope spectroscopy of PG $1605+072$. The $1 / f$ component of the noise is low because of our removal of slow drifts. The final white noise level is $207 \mathrm{~m} \mathrm{~s}^{-1}$.

not just an average over the whole night. Also, since variations are visible in many parts of the velocity curve (see the bottom panel of Fig. 1), the real variation may be confused with noise. Finally, data that lie outside $\pm 100 \mathrm{~km} \mathrm{~s}^{-1}$ - where a sharp drop in $\mathrm{S} / \mathrm{N}$ was caused by passing clouds - were weighted significantly lower than those within.

Despite the regular taking of arc spectra, there are still drifts and jumps in the velocity curve, especially in the observations made with grism spectrographs. To remove these, we firstly did a crude analysis, whereby we fit sinusoids to and subtracted them from each night's data until all that remained were the white and $1 / f$ noise components. To the signal-free residuals, we fit a series of polynomials of degree $1-3$, and then subtracted the fits from the original raw velocity curve. The trends we remove are non-periodic, and caused by instrumental effects; they are most likely not intrinsic to the star. The final velocity curve with trends removed is shown in the middle panel of Fig. 1. This is a significant improvement on our initial velocity curve, increasing the possibility of detecting low amplitude oscillations. The bottom panel of Fig. 1 shows a section of the velocity curve from the second night of observations (20 May 2002) at Steward Observatory; velocity variations with a period around $480 \mathrm{~s}$ are evident between 1.76 and 1.78 days.

The velocity amplitude spectrum was calculated using a weighted Fourier transform and is shown in Fig. 4. We determined the noise level in the spectrum in two ways: by averaging the amplitude spectrum in regions where no signal exists (i.e. at high frequencies); and by fitting an exponential function to the prewhitened spectrum. The former method is only an indication of the white noise level, which we find to be $207 \mathrm{~m} \mathrm{~s}^{-1}$, while the latter method includes an approximation of the $1 / f$ component as well. The noise function we derived is shown in Fig. 5. Because of the removal of slow drifts, the $1 / f$ noise is virtually the same as the white noise in the frequency range of interest. The noise level is almost $45 \%$ lower than in O'Toole et al. (2002).

\section{Frequency analysis}

The frequencies presented here were determined using Period98, a least-squares fitting program (Sperl 1998) that fits multiple sinusoids simultaneously with the following form:

$Z+\sum_{i=0}^{N} A_{i} \sin \left(2 \pi f_{i} t+\phi_{i}\right)$

where $Z$ is the zero point shift, $A_{i}$ is the amplitude, $f_{i}$ is the frequency and $\phi_{i}$ is the phase. The analysis was performed in an iterative manner, and the amplitude of each peak was compared with the noise function discussed earlier. If the peak is four times the noise then we consider it to be real. There are two exceptions to this, however: peaks at $3967.29 \mu \mathrm{Hz}$ and $3993.88 \mu \mathrm{Hz}$ are considered real because of their very good correspondence to linear combinations of higher amplitude modes. Our full list of frequencies is shown in Table 3.

\subsection{Below $2500 \mu \mathrm{Hz}$}

Most of the oscillation power of PG $1605+072$ lies in the frequency range $1800-2500 \mu \mathrm{Hz}$. The dominant peak in the amplitude spectrum, as seen in the Fig. 4 , lies at $2075.80 \mu \mathrm{Hz}\left(f_{1}\right)$. In earlier velocity studies (O'Toole et al. 2000, 2002; Woolf et al. 2002b) this frequency has been found to have a much 
Table 3. Frequencies, velocity semi-amplitudes and periods from the entire $2 \mathrm{~m}$ campaign; $n$ is the amplitude rank. Entries marked with an asterisk (*) have amplitudes below our threshold of four times the white noise level, however their correspondence with combination frequencies is good enough to suggest their reality.

\begin{tabular}{cccccc}
\hline \hline$n$ & $\begin{array}{c}f \\
(\mu \mathrm{Hz})\end{array}$ & $\begin{array}{c}V \\
\left(\mathrm{~km} \mathrm{~s}^{-1}\right)\end{array}$ & $\begin{array}{c}P \\
(\mathrm{~s})\end{array}$ & $\begin{array}{c}\text { Freq. } \\
\text { sums }\end{array}$ & $\begin{array}{c}\Delta \\
(\mu \mathrm{Hz})\end{array}$ \\
\hline 6 & 1891.41 & 2.322 & 528.71 & & \\
5 & 1985.89 & 2.474 & 503.55 & & \\
1 & 2075.80 & 15.429 & 481.74 & & \\
3 & 2101.91 & 2.971 & 475.76 & & \\
2 & 2102.55 & 5.372 & 475.61 & & \\
14 & 2201.20 & 0.977 & 454.30 & & \\
17 & 2202.18 & 0.919 & 454.10 & & \\
13 & 2270.37 & 0.998 & 440.46 & & \\
18 & 2738.42 & 0.901 & 365.17 & & \\
4 & 2743.01 & 2.497 & 364.56 & & \\
11 & 2762.67 & 1.155 & 361.97 & & \\
7 & 2763.50 & 2.121 & 361.86 & & \\
9 & 2764.45 & 1.655 & 361.74 & & \\
15 & 2842.94 & 0.967 & 351.75 & & \\
12 & 2844.59 & 1.112 & 351.54 & & \\
16 & 2852.31 & 0.931 & 350.59 & & +0.02 \\
22 & $3967.29^{*}$ & 0.788 & 252.06 & $f_{1}+f_{6}$ & +0.08 \\
21 & $3993.88^{*}$ & 0.810 & 250.38 & $f_{2}+f_{6}$ & -0.08 \\
8 & 4061.70 & 1.777 & 246.20 & $f_{1}+f_{5}$ & -0.01 \\
10 & 4151.62 & 1.364 & 240.87 & $2 f_{1}$ & +0.02 \\
19 & 4178.36 & 0.858 & 239.33 & $f_{1}+f_{2}$ & +0.01 \\
20 & 6137.56 & 0.845 & 162.93 & $2 f_{1}+f_{5}$ & +0.07 \\
\hline & & & & &
\end{tabular}

reduced amplitude compared to that seen in intensity by both Koen et al. (1998) and Kilkenny et al. (1999), although the most recent study prior to our observations found that it was dominant again (Falter et al. 2003). This apparently variable amplitude may be intrinsic to the star, or may simply be caused by beating between two or more very closely spaced peaks. Since these possibilities are difficult to explain theoretically, we leave both open for further investigation.

The peaks found at $2101.90 \mu \mathrm{Hz}$ and $2102.55 \mu \mathrm{Hz}$ match quite well those found by O'Toole et al. (2002), although there is a small frequency difference. O'Toole et al. found an extra frequency at $2102.83 \mu \mathrm{Hz}$, probably because of their better frequency resolution. These frequencies were not resolved by the other three velocity studies of PG $1605+072$. The multisite photometric study of Kilkenny et al. (1999) found the two peaks, but at slightly different frequencies, and different again to the frequencies measured by O'Toole et al. (2002). The reason for these apparent frequency differences, as well as the variation of the $2075.8 \mu \mathrm{Hz}$ peak, is uncertain, although they resemble the stochastically excited oscillations found in the Sun and solar-like stars. A recent study by Pereira \& Lopes (2005) seems to rule this out however. This is perhaps not surprising, since solar-like oscillations are related to stellar convection zones in the atmosphere, and sdBs are not predicted to show such zones. The nature of this amplitude variability remains unknown; it seems likely to us that it is the result of the damping and re-excitation.

We now comment briefly on the other modes detected in this region.

- The peak at $2202.18 \mu \mathrm{Hz}$ was actually detected at $\sim 2190.5 \mu \mathrm{Hz}$, however we believed this to be separated from the true frequency by an alias. Kilkenny et al. (1999) found a peak in their photometry at $\sim 2201.93$, so we have fit $2190.5+11.67 \mu \mathrm{Hz}$ instead.

- Kilkenny et al. (1999) measured the peak at $1985 \mu \mathrm{Hz}$ to have the 8th highest amplitude, however, in subsequent studies its amplitude relative to the other peaks is consistently higher. For example, Woolf et al. (2002b), O'Toole et al. (2002) and Falter et al. (2003) detected the mode, while O'Toole et al. (2000) did not.

- The $1891 \mu \mathrm{Hz}$ peak was detected by O'Toole et al. (2000), Woolf et al. (2002b) and O'Toole et al. (2002), but not by Falter et al. (2003), although in Fig. 3 of the latter study, the peak is possibly present, but the low frequency resolution and $\mathrm{S} / \mathrm{N}$ makes it difficult to be certain.

- The mode at $\sim 2270 \mu \mathrm{Hz}$ has only previously been marginally detected by O'Toole et al. (2002) - our detection is much more confident. This mode may form part of a rotationally split triplet (Kawaler 1999, see Sect. 4).

There is still an excess of power in the $2000-2200 \mu \mathrm{Hz}$ range, in particular around $\sim 2085 \mu \mathrm{Hz}$ and $\sim 2167 \mu \mathrm{Hz}$. An excess also exists around $1650 \mu \mathrm{Hz}$, however no pulsation frequency has ever been measured in that range for PG 1605+072 before. While we are certainly not claiming these as detections, it is encouraging that the first two frequencies correspond well to frequencies measured by Kilkenny et al. (1999). Full analysis of our photometry may clarify the reality of these peaks, although perhaps not if they have $\ell>2$.

\section{2. $2500-3500 \mu \mathrm{Hz}$}

The strongest mode in this range is at $2743.01 \mu \mathrm{Hz}$, and was the strongest mode overall in the studies of O'Toole et al. (2002) and Woolf et al. (2002b), although in both these studies it was split in two (by quite different amounts). This has already been discussed by O'Toole et al. No such splitting is seen in our data, suggesting again that the mode amplitudes are varying with time. This idea is supported by several low amplitude modes found nearby by Kilkenny et al. (1999).

The modes around $2763 \mu \mathrm{Hz}$ have been previously detected in photometry, although Kilkenny et al. (1999) found them only after observations additional to their main campaign were included in the analysis. Otherwise they list two frequencies at 2761.3 and 2766.3 in their Table 3. A peak at $2764.1 \mu \mathrm{Hz}$ was detected by Koen et al. (1998) which matches our detection well considering their very complicated window function. As before, these modes could be the result of damping and reexcitation of one pulsation mode.

We have detected two peaks around $2843 \mu \mathrm{Hz}$ for the first time in velocity, although they have been observed before by Kilkenny et al. (1999). The lower frequency of the 


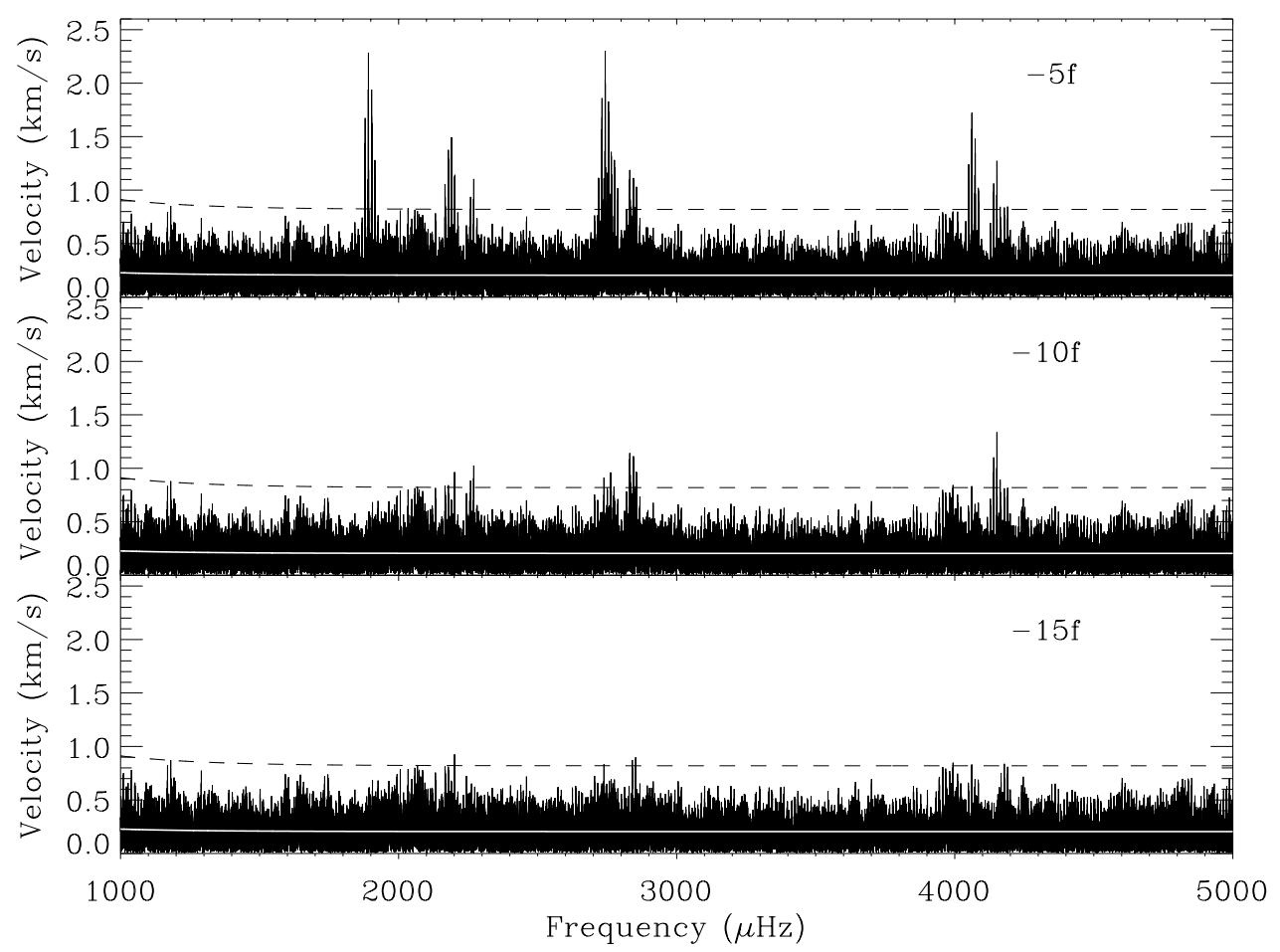

Fig. 5. Amplitude spectrum after prewhitening. The white line shows the noise function determined in Sect. 3, while the dashed line represents our detection threshold, or four times the noise function.

pair was originally detected at $2831.28 \mu \mathrm{Hz}$, however because of the Kilkenny et al. detection separated by approximately one cycle-per-day, we believe this to be the correct value. These frequencies may form part of a multiplet (see Sect. 4).

There are two frequencies we have detected at $2738.42 \mu \mathrm{Hz}$ and $2852.31 \mu \mathrm{Hz}$, which correspond approximately to peaks found by Koen et al. (1998) in photometry, but not seen by Kilkenny et al. (1999). Again, a full analysis of our simultaneous photometry should help to clarify the status of these peaks.

\subsection{Above $3500 \mu \mathrm{Hz}$}

In this range the frequencies we have detected are linear combinations of the frequencies below $2500 \mu \mathrm{Hz}$ discussed in Sect. 3.1.

We have detected the first harmonic of the highest amplitude peak in our observations $\left(f_{1}\right)$; this is the first detection of this kind in velocity, although Thompson et al. (2003) measured a combination frequency in a velocity study of the DAV white dwarf G29-38. Schrijvers \& Telting (1999) considered line-profile variations due to non-radial adiabatic pulsations in rotating stars. They found that any oscillations where temperature effects dominate are not expected to show detectable harmonic variability in the line profile at all. Our detection therefore suggests that line profile variations play a significant role. More theoretical work is needed in this area.

The five other frequencies above $3500 \mu \mathrm{Hz}$ found in Table 3 are linear combinations of the strongest modes. This is first time that the second order linear combination $2 f_{1}+f_{5}$ has been detected in PG $1605+072$, although its frequency $(6137.56 \mu \mathrm{Hz})$ is not the highest that has been detected in this
Table 4. Relationships between phases of combination frequencies and their parent modes. Phases have been normalised to the range $[-\pi, \pi]$.

\begin{tabular}{ccccc}
\hline \hline$f_{i}+f_{j}\left(f_{k}\right)$ & $\phi_{i}$ & $\phi_{j}$ & $\phi_{k}$ & Relation \\
& $\left({ }^{\circ}\right)$ & $\left({ }^{\circ}\right)$ & $\left({ }^{\circ}\right)$ & \\
\hline $2 f_{1}\left(f_{10}\right)$ & $-35 \pm 1$ & - & $94 \pm 9$ & $2 \phi_{1} \approx \pi+\phi_{10}$ \\
$f_{1}+f_{2}\left(f_{19}\right)$ & $-35 \pm 1$ & $31 \pm 2$ & $164 \pm 13$ & $\phi_{1}+\phi_{2}$ \\
& & & & $\approx \pi+\phi_{19}$ \\
$f_{1}+f_{5}\left(f_{8}\right)$ & $-35 \pm 1$ & $158 \pm 5$ & $57 \pm 7$ & $\phi_{1}+\phi_{5}$ \\
& & & & $\approx \pi-\phi_{8}$ \\
$2 f_{1}+f_{5}\left(f_{20}\right)$ & $-35 \pm 1$ & $158 \pm 5$ & $-169 \pm 14$ & $\phi_{1}-\phi_{5} \approx-\phi_{20}$ \\
$f_{1}+f_{6}\left(f_{22}\right)$ & $-35 \pm 1$ & $156 \pm 5$ & $-121 \pm 15$ & $\phi_{1}+\phi_{6} \approx-\phi_{22}$ \\
$f_{2}+f_{6}\left(f_{21}\right)$ & $31 \pm 2$ & $156 \pm 5$ & $-170 \pm 14$ & $\phi_{2}+\phi_{6} \approx \phi_{21}$ \\
\hline
\end{tabular}

star. Koen et al. (1998) detected frequencies up to $\sim 8400 \mu \mathrm{Hz}$, although these frequencies were not detected in the multisite observations of Kilkenny et al. (1999). All other combination frequencies are first order, and have been detected in photometry by earlier studies.

When we examine the phases of the combination frequencies and their parent modes, we find a few interesting results, although we remain cautious about any possible interpretation. The relationships are shown in Table 4. Phase errors are determined in complex phase space. What these relations say about the character of the modes is unclear without theoretical modelling. Wu (2001) investigated combination frequencies in pulsating DA and DB white dwarfs and derived analytic expressions for their amplitudes and phases. Wu also found that 
mode identification is possible using combination frequencies. Unfortunately we cannot apply the results of her analysis here, since pulsating white dwarfs have convection zones while sdBs do not, but the potential is exciting nevertheless.

\section{Further discussion}

Because of the large number of modes observed in PG 1605+072 and its relative brightness, it has become one of the most studied sdB stars.

Kawaler (1999) found a model matching the 1891, 2075 and $2270 \mu \mathrm{Hz}$ peaks found by Kilkenny et al. (1999) to an $l=1$ triplet. From this he derived a rotation velocity of $\sim 130 \mathrm{~km} \mathrm{~s}^{-1}$; Heber et al. (1999) later measured $v \sin i=$ $39 \mathrm{~km} \mathrm{~s}^{-1}$ from high resolution spectroscopy, a value that is consistent with Kawaler's determination if the inclination angle of the star is $\sim 17^{\circ}$. We have observed each of these modes in our velocity amplitude spectrum for the first time.

Interestingly, in Table 3 there are three pairs of frequencies $-(2101.90,2201.20) \mu \mathrm{Hz},(2102.55,2202.18) \mu \mathrm{Hz}$ and (2742.99, 2842.95) $\mu \mathrm{Hz}$ - separated by $\sim 100 \mu \mathrm{Hz}$. There are further pairs that can be seen in Kilkenny et al. (1999) at $(1985.32,2085.84) \mu \mathrm{Hz}$ and $(2842.05,2942.77) \mu \mathrm{Hz}$. The first peak of the former pair is present in our amplitude spectrum and as discussed in Sect. 3.1, there is evidence for a lowamplitude peak at $\sim 2085 \mu \mathrm{Hz}$. One must be careful not read too much into these patterns without analysis of our photometry, however!

\section{Summary and conclusions}

We have presented observations and analysis of the $2 \mathrm{~m}$ spectroscopic observations of the pulsating sdB star PG 1605+072 from the MSST campaign. With more than $150 \mathrm{~h}$ and almost 11000 spectra, we have been able to reduce our white noise level to $207 \mathrm{~m} \mathrm{~s}^{-1}$, allowing the detection of the largest number of frequencies in velocity ever in an sdB. Among these are five first order and one second order linear combinations; this is only the second time that such frequencies have been observed in velocity. The highest amplitude mode from the discovery observations is once again dominant, after several seasons of low amplitudes or even non-detections.

Since such a large amount of information can be extracted from this data set - for example a line index study similar to O'Toole et al. (2003) - we have decided to make it available for public use (please contact the authors). This will also eventually include our $4 \mathrm{~m}$ spectroscopy and our almost $400 \mathrm{~h}$ of photometry. With the combination of all of these data, asteroseismology may begin to unravel one of the most complex pulsating stars known.

Acknowledgements. We are grateful to the various time allocation commitees who gave generously. S.J.O.T. was partially supported by an Australian Postgraduate Award and is currently supported by the Deutsches Zentrum für Luft- und Raumfahrt (DLR) through grant No. 50-OR-0202. V.M.W. was supported by the UK Particle Physics and Astronomy Research Council (PPA/G/S/1998/00019 and PPA/G/O/2001/00068). C.S.J. acknowledges support to the Armagh Observatory from the Northern Ireland Dept. of Culture, Arts and Leisure. We would also like to thank Tim Bedding for kindly providing support and funding for observers to stay at SSO.

\section{References}

Brassard, P., Fontaine, G., Billères, M., et al. 2001, ApJ, 563, 1013 Charpinet, S., Fontaine, G., \& Brassard, P. 2001, PASP, 113, 775

Dorman, B., Rood, R. T., \& O'Connell, R. W. 1993, ApJ, 419, 596

Falter, S., Heber, U., Dreizler, S., et al. 2003, A\&A, 401, 289

Fontaine, G., Brassard, P., Charpinet, S., et al. 2003, ApJ, 597, 518

Green, E. M., Fontaine, G., Reed, M. D., et al. 2003, ApJ, 583, L31

Han, Z., Podsiadlowski, P., Maxted, P. F. L., \& Marsh, T. R. 2003, MNRAS, 341, 669

Heber, U. 1986, A\&A, 155, 33

Heber, U., Dreizler, S., Schuh, S. L., et al. 2003, in 13th European Workshop on White Dwarfs, ed. D. de Martino, R. Silvotti, J.-E. Solheim, \& R. Kalytis (Kluwer), 105

Heber, U., Reid, I. N., \& Werner, K. 1999, A\&A, 348, L25

Jeffery, C. S., Dhillon, V. S., Marsh, T. R., \& Ramachandran, B. 2004, MNRAS, 119

Jeffery, C. S., \& Pollacco, D. 2000, MNRAS, 318, 974

Kawaler, S. D. 1999, in 11th. European Workshop on White Dwarfs, ed. J. E. Solheim, \& E. G. Meistas, ASP Conf. Ser., 169, 158

Kilkenny, D. 2002, in IAU Colloq., 185: Radial and Nonradial Pulsations as Probes of Stellar Physics, ASP Conf. Ser., 259, 356

Kilkenny, D., Koen, C., O’Donoghue, D., et al. 1999, MNRAS, 303, 525

Koen, C., O’Donoghue, D., Kilkenny, D., et al. 1998, MNRAS, 296, 317

O’Toole, S. J., Bedding, T. R., Kjeldsen, H., Dall, T., \& Stello, D. 2002, MNRAS, 334, 471

O’Toole, S. J., Bedding, T. R., Kjeldsen, H., et al. 2000, ApJ, 537, L53

O'Toole, S. J., Jørgensen, M. A. S. G., Kjeldsen, H., et al. 2003, MNRAS, 340, 856

Pereira, T. M. D., \& Lopes, I. P. 2005, ApJ, 622, 1068

Schrijvers, C., \& Telting, J. H. 1999, A\&A, 342, 453

Sperl, M. 1998, Available for download from ftp://dsn . astro. univie.ac.at/pub/Period98/

Telting, J. H., \& Østensen, R. H. 2004, A\&A, 419, 685

Thompson, S. E., Clemens, J. C., van Kerkwijk, M. H., \& Koester, D. 2003, ApJ, 589, 921

Woolf, V. M., Jeffery, C. S., \& Pollacco, D. 2003, in 13th European Worskshop on White Dwarfs, ed. D. de Martino, R. Silvotti, J.-E. Solheim, \& R. Kalytis (Kluwer), 95

Woolf, V. M., Jeffery, C. S., \& Pollacco, D. L. 2002a, MNRAS, 332, 34

Woolf, V. M., Jeffery, C. S., \& Pollacco, D. L. 2002b, MNRAS, 329, 497

Wu, Y. 2001, MNRAS, 323, 248 